Review

\title{
Recent Advances in Nanofabrication of the Morphology-Controlled Hypercrosslinked Polymers
}

\author{
Wenliang Song ${ }^{*}$, Mingxin Zhang, Mingjie Liu, Yinning Huang, Dengguang $\mathrm{Yu}^{*}$ \\ School of Materials Science \& Engineering, University of Shanghai for Science and Technology, 516 Jun-Gong Road, Shanghai 200093, China
}

\section{ARTICLE INFO}

\section{Article History}

Received 28 April 2021

Accepted 08 June 2021

Keywords

Nanofabrication

hypercrosslinked polymers

morphology-control

\begin{abstract}
Hypercrosslinked Polymers (HCPs) have received considerable attention in the field of catalysis, adsorption, separation, biomedical engineering, and energy storage/conversion, because of their permanent microporous structures and high surface areas. Up to now, various "bottom-up" and "top-down" methods have been reported to synthesize of the HCPs with diverse morphologies, and these morphologies have been demonstrated to play a significant role in various applications. In this review, we summarized the latest research in the design of morphology-controlled HCPs by using three kinds of main methods and we also emphasized on the template free solutions. Furthermore, the future developments and challenges for fabricating of morphology-controlled HCPs are also presented in this review.
\end{abstract}

(C) 2021 The Authors. Published by Atlantis Press B.V.

This is an open access article distributed under the CC BY-NC 4.0 license (http://creativecommons.org/licenses/by-nc/4.0/)

\section{INTRODUCTION}

Hypercrosslinked Polymers (HCPs) have obtained considerable research interest because of their combine many advantages such as well-defined porosity, high surface area, lightweight nature, and tunable branched surface groups in one [1,2]. Commonly, the HCPs synthetization have three main approaches: (1) postcrosslinking polymers precursors, (2) direct polycondensation, and (3) knitting by external crosslinkers [3,4]. A variety of monomers, aromatic building blocks and the external crosslinkers, coupled with these synthetic approaches makes the HCPs become invaluable platforms for exploring new advanced porous materials [5-7]. And these methods endow these polymers with diverse inner porosity and outer morphologies (Figure 1). On this basis, some unique properties are revealed on HCPs, and a wide range of practical and potential applications, such as catalysis [8-10], adsorption [11-14], separation, and energy storage/conversion [15-17], are also reported by these researchers [8,11-17].

The outer shapes of porous materials have been reported could direct or indirectly affect their features and properties. Some reports also shown the morphologies could influence the performance of these porous polymers, their carbonaceous counterparts, and inorganic/polymer hybrid materials [18]. For example, Zhang et al. [19] showed that dimensionality could significantly influence on the photoluminescence properties of porous polymers. Also, Jiang et al. reported a novel method for fabricating of polymeric tubes. When compared with the spherical carbon electrode materials, polymeric precursors with tubular morphologies could

"Corresponding authors.Email: wenliang@pusan.ac.kr; ydg017@usst.edu.cn significantly enhance the stability of the supercapacitors as the carbon electrodes [20]. Son et al. reported the multishelled hollow morphologies could enhance the drug loading and release efficiency. As the shell number increased, the drug loading and release efficiency increased, because the intershell space have been proved with great influence on the drug delivery properties [21]. From the reported literatures, the morphology of HCPs could play a unique role in various applications [22].

This review will discuss the progress of the research on the morphology control of HCPs. Wherever needed, we shall compare unique morphology-controlled HCPs with normal one and invoke the performances of polymer nanoparticles in applications to highlight the similarities and differences. Toward the end, the unresolved issues, and future directions of research on the morphology-control of HCPs will be deliberated.

\section{CLASSIFICATIONS}

Hypercrosslinked polymers particles are morphologically into four types, the Zero-dimensional (0D) microspheres, 1D nanotubes/fibers, 2D nanosheets/films, and 3D monoliths/flowers. The spheres also could be divided into hollow and solid one. Not only these types, some hybrid morphologies and some unconventional shapes also been reported [23,24], for example, the preparation of a three-layer core-shell structure of Fe/Fe $\mathrm{O}_{4} @ \mathrm{TCNFs} @ \mathrm{TiO}_{2}$ magnetic hybrid nanofibers reported by Zhang et al. As shown in Figure 2, these diverse morphologies are generally fabricated by using three main methods: (1) Template methods (hard template and soft template), (2) Template-free method, and (3) Physicalmechanical method. 


\subsection{Template Method}

Template method is the most straightforward method for generating the morphology-controlled HCPs. Template method could be divided into hard template and soft template method. The hard template method generally contains three steps: Firstly, the uniformed hard templates are prepared by the scalable method. Secondly, follow the common synthetic approaches, such as hydrothermal method and sol-gel method, the hybrid polymers are prepared to generate the materials that coexisted with the template. The hyper crosslinking is processed during or after this procedure. Finally, these templates are removed by the suitable method to avoid the damage to the targeted materials, in some reported research, the hard template also coexisted with these polymers [25]. In the template method for fabricating of the HCPs, we should precisely choose a suitable template with the ordered and qualified shapes. Thus far, various materials such as silica [26-28], graphene [29], $\mathrm{Fe}_{3} \mathrm{O}_{4}$ [30,31], gold [32], and $\mathrm{ZnO}$ [33] are all used as the hard template to control the morphologies of HCPs. The

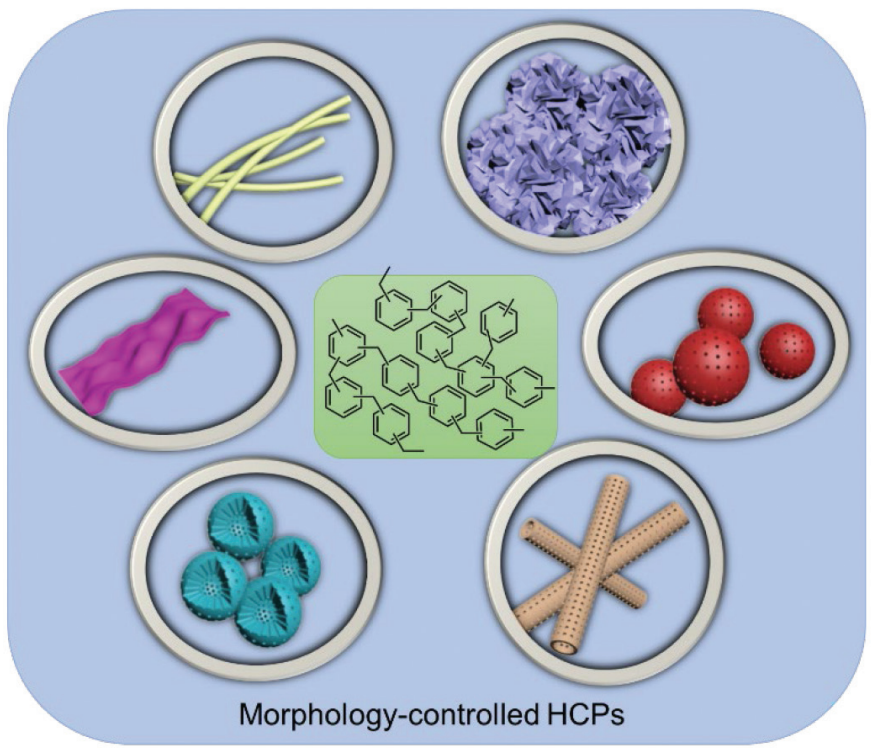

Figure 1 Summary of the various morphologies generated in the HCP samples. hollow structure is the most fascinating morphologies. The sacrificing and etching of $0 \mathrm{D}$ and $1 \mathrm{D}$ templates can directly generate the hollow morphologies with the controlled inner shape and size, meanwhile, the shell can protect the inner space from the outer environment. Tan et al. firstly reported synthesizing of the hollow spheres and hollow rods by using the template method (Figure 3a). The spherical and rod-liked silica were firstly synthesized and modified with characteristic groups, the polymerization and crosslinking process were further induced to the surface of modified silica $[27,28]$. The spherical and rod-liked HCPs were generated by etching with the HF to remove the silica template, main problems with this method are the waste of hard templates, and the possible danger in the use of toxic HF acid. These void and hollow spaces inside of the HCPs structures could accommodate guest compounds, increase the diffusion rate, and give surface functionality. So, HCPs with hollow morphologies could be used in various applications, such as nanoreactors, energy storage materials, and reaction catalysts. Also, Tan et al. [34,35] examined the performances of these synthesized hollow particles as the platform for various applications, such as catalysts, sensors, and drug carriers. The hollow HCPs process multiply functionality and could be functionalized as the effective acid-base catalysts (Figure 3b) [36]. Not only silica, Son also showed the hollow spheres with thin shells could directly utilized as the template for engineering the HCPs. Further, the HCPs with BINOLs phosphoric acid could directly be deposited on this template, and they showed good catalytic performance as a heterogeneous catalytic system [37], when H-CMPL@ HCP-BP was used as the catalyst, the yield of PCLs at $150^{\circ} \mathrm{C}$ could reach $97 \%$. The hollow HCPs precursors also could be transferred to the hollow porous carbon spheres by pyrolyzing at the inert condition. This method also be used to generate the hollow hybrid HCPs materials, for example, Tan et al. [38] reported the hollow HCPs fabricated with $\mathrm{NH}_{2}$ groups were synthesized and used as template for Pt nanoparticles. This hybrid systems exhibit excellent catalytic activity and recyclability potential. Following this template approach, Wu et al. [39] prepared crosslinked polystyrene spheres, the carbonyl bridges between polystyrene nanoparticles forms unique network-type microporosity. The $\mathrm{Fe}_{3} \mathrm{O}_{4}$ supraparticle is an important template that no need to be removed, Guo et al. prepared the nanoscale porous organic polymer composite which consisting of $\mathrm{Fe}_{3} \mathrm{O}_{4}$ nanoparticles as the core and porous polymer as the outer shell. In this approach, the $\mathrm{Fe}_{3} \mathrm{O}_{4} @ \mathrm{PS}$ microspheres
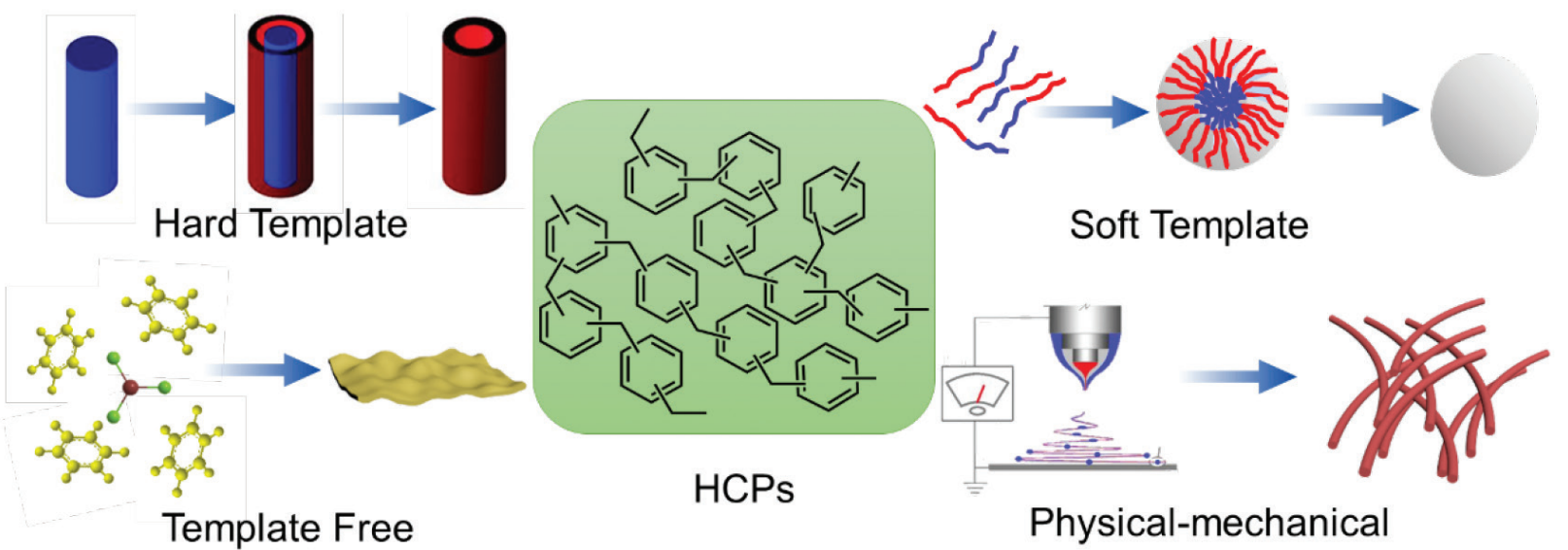

Figure 2 Summary of the general methods for controlling of the HCP morphologies. 


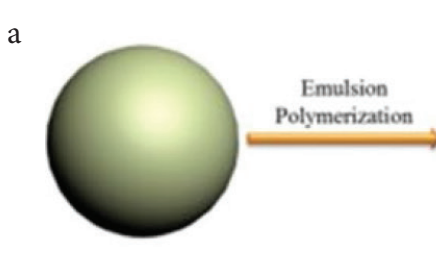

$\mathrm{SiO}_{2} \mathrm{NPs}$

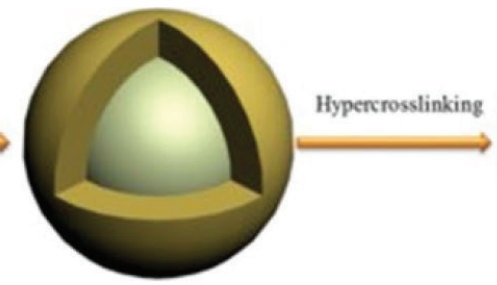

$\mathrm{SiO}_{2} @$ PS-DVB

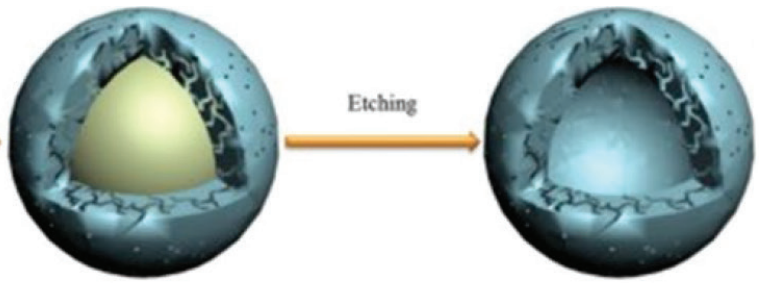

Hypercrosslinked $\mathrm{SiO}_{2} @$ PS-DVB
Hollow Microporous Organic Capsules (HMOCs) b

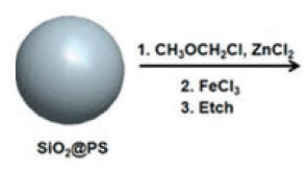

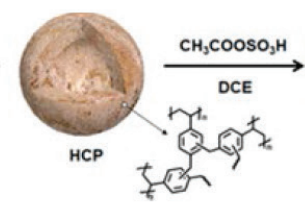

Ho,s
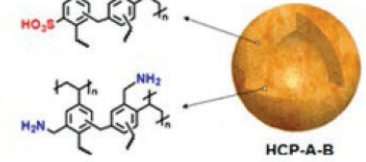

1. $\mathrm{NaHCO}_{3}$

2. $\mathrm{NH}_{2} \mathrm{NH}_{2} \cdot \mathrm{H}_{2} \mathrm{O}$

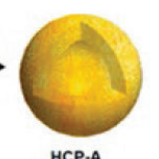

HCP.A

NHPI, TFA

TFOH/DCM

c
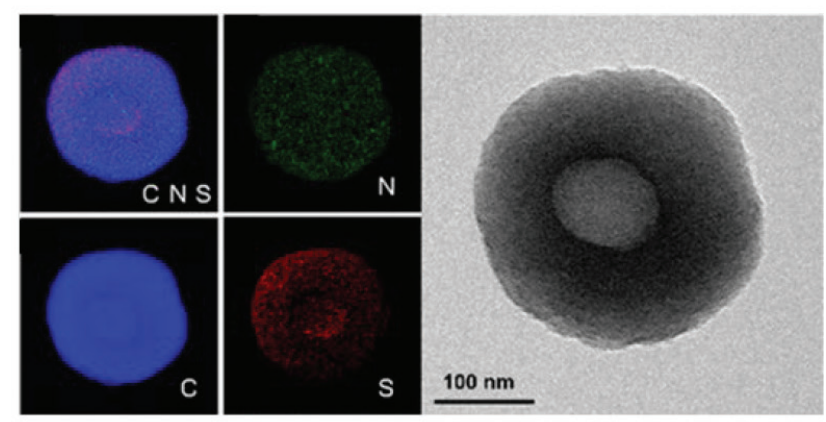

Figure 3 Hard template method for fabricating of the morphology-controlled HCPs. (a) Scheme for the fabrication of the hyper crosslinked hollow spheres [27]. Copyright 2015. Adapted with permission from Springer Nature. (b) Scheme for the fabrication of the acid base bifunctionalized hyper crosslinked hollow spheres. (c) Energy dispersive X-ray analysis (EDX) mapping results of the bifunctionlized hollow hyper crosslinked hollow spheres [36]. Copyright 2008. Adapted with permission from the American Chemical Society.

are firstly synthesized, after then the poly[Vinylbenzyl Chloride (VBC)-co-Divinylbenzene (DVB)] network were blended with the Polystyrene (PS) shell. By varying the feed ratio of the VBC and DVB monomer in the hyper cross-linking treatment, the mesopores could tuned from 4 to $30 \mathrm{~nm}$, these precisely adjustable channels could be used to confine the platinum metal (Pt) nanoparticles. Therefore, the final composite shows excellent catalytic activities in the hydrogenation applications [30].

In conclusion, the hard template is the most direct solution to form the hollow morphologies, but this method is complex and inflexible. In some related approach, hard template no need to be removed, and will coexist with the HCP polymer frameworks. For example, Pandey et al. reported the $\mathrm{Fe}_{3} \mathrm{O}_{4}$ could be used as the hard template, the VBC could be deposited on this template, after further amination, the highly cross-linked hybrid materials could be generated. In this approach, the encapsulated $\mathrm{Fe}_{3} \mathrm{O}_{4}$ nanoparticles retained super paramagnetic properties, and hydrated anions were absorbed in this hybrid hydrophobic polymers [31]. Compared with other approach, hard template no need post removal and coexisted with these polymers in this method, which presented an important role for generating the morphology-controlled hybrid HCPs, but their types are often limited by the hard templates.

Compared with the hard template method, the soft template is easier to be removed or coexisted with the final products. By using the surfactants, biological molecules, and synthetic polymers, the self-assembly occurs during or after the polymerization process. $\mathrm{Wu}$ et al. reported a facile method for generating the $\mathrm{pH} / \mathrm{tem}$ perature responsible, hyper crosslinked polymeric spheres. The hairy functional blocks were growing on the polymer spheres by utilizing the surface-initiated atom transfer radical polymerization method [40]. The suspension polymerization method is often used in the soft template approach. Wu et al. showed the monodisperse, hyper-crosslinked poly(4-chloromethylstyrene) nanospheres (xPCMS) could further be functionalized with carbazole dithioester and maleic anhydride, the soft template could be introduced to the surface of spheres to produce the poly(2-(dimethylamino) ethyl methacrylate) shell by Reversible Addition-Fragmentation Chain Transfer (RAFT) polymerization. This all-in-one porous polymer adsorbents showed unimpeded adsorption performance, the visible fluorescence quenching could be used for detecting the pollutants, also, the organic micropollutants could be removed with super-rapid speed [41], xPCMS exhibited rapid organic micropollutant adsorption ( $98 \%$ and $100 \%$ of its equilibrium uptake within $10 \mathrm{~s}$ and $2 \mathrm{~min}$, respectively).

Huang and Rzayev firstly reported the bottlebrush copolymers could self-assembly into cylindrical shapes in solutions. Following the cross-linking method, the shapes and size of original bottlebrush macromolecules could be preserved after etching of the core (Figure $4 \mathrm{a}$ and $4 \mathrm{~b}$ ). In this approach, the hierarchical organic polymeric tubular networks with micro-, meso- and macroporous pores could be directly obtained [42]. The various functional groups, such as amine, amino, sulfonic acid, porphyrin, and thiol could be in situ or post fixed to the tubular networks by this soft template approach [43,44]. Huang et al. [45] also showed that the Fe and Oxo-vanadium could supported by this microporous organic nanotube. After then, Wu et al. firstly reported using of the self-assembly and hyper crosslinking method for generating the hollow polymeric spheres. The poly (methyl methacrylate)- $b$-polystyrene diblock copolymer was first self-assembled into uniformed core-shell spheres, and further transform to the hollow carbon nanospheres by the followed hyper crosslink process (Figure 5) [46]. The design of the block copolymer and the interaction with the catalyst are not simple. Also, Huang et al. reported the polylactide- $b$-polystyrene could be used for fabricating of the hollow 
a

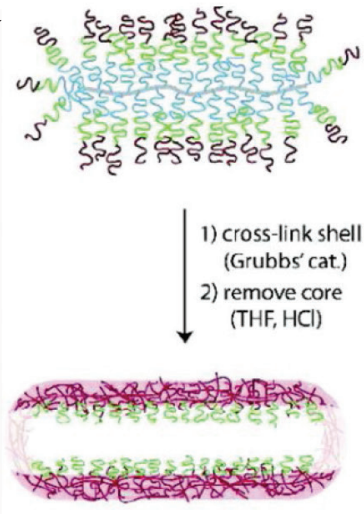

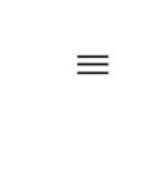
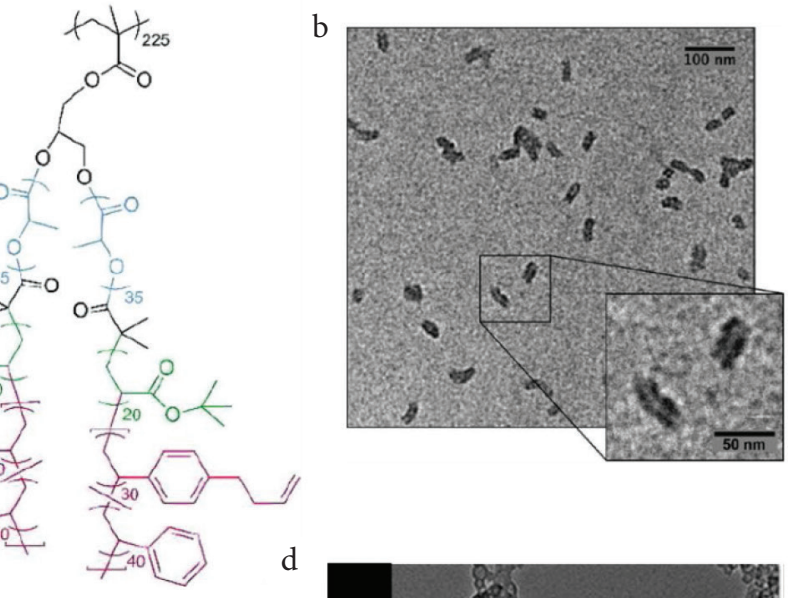

\section{d}

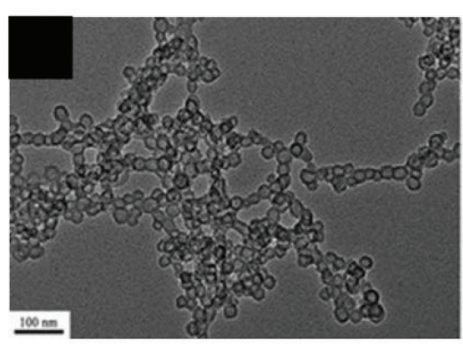

Figure 4 Soft template method for fabricating of the morphology-controlled HCPs. (a) Scheme for the fabrication of the hyper crosslinked tubes by using the bottlebrush copolymers. (b) Transmission Electron Microscope (TEM) analysis of the hyper crosslinked tubes [42]. Copyright 2011. Adapted with permission from the American Chemical Society. (c) Scheme for the fabrication of the hyper crosslinked hollow spheres by using the block copolymers; (d) TEM analysis of the hyper crosslinked hollow spheres [24]. Copyright 2017. Adapted with permission from the American Chemical Society.

polymer spheres (Figure 4a and 4b). Unlike the self-assembly mechanism produced by Wu et al. Huang proposed the byproduct $\mathrm{HCl}$, which generated in the Friedel-Crafts reactions, could change the dissolution capability of polylactide chain, thus the spherical micelle-like intermediate could be generated, after the polylactide be degraded, the hollow polymer spheres were eventually formed [24]. From the above research results, we can conclude the composition of blocked or branched polymer chains play a key role on the morphology forming of the HCPs. Not only the composition, but the solvents also can induce the morphologies transformation, Qiao et al. showed the polarity of the solvent has a dramatic effect upon the oleophobic/oleophilic interaction. The final morphologies could tailor from the aggregated particles to hollow spheres and even the mesoporous bulk by simply adjusting of the solvent composition. The hollow morphologies give the cavity inside of the spheres, which could be used as containers or reactors [47]. During the solution state, the micropores could swell in concert with the mesopores, although the reactant can penetrate through the micropores of porous skeleton, but the mesopores could dramatically shorten the diffusion pathway. So that the polymers with mesopores, especially the nanotubes and nanospheres with small sizes and ordered channels often show compatible activities than the corresponding disordered polymers [48].

Nanosheets are another interesting morphology that could be generated by the polymers. Commonly, these polymeric shapes are difficult to be obtained by template method, Zhuang et al. show the trithiocarbonate functionalized graphene oxide could be used as $2 \mathrm{D}$ raft agent. The poly(VBC-co-DVB) precursor resin was grafted, the hyper crosslinking reaction was carried out for benzyl chloride groups, and then the well-defined $2 \mathrm{D}$ nanosheets were generated [29]. Compared with unadorned HCPs obtained without the graphene template, these polymers exhibited improved thermal stability, high surface area, and could be used as carbon precursor for energy storage. Another soft chemistry synthetic strategy based on the Friedel-Crafts alkylation is developed for the phenolic resin. This resin with uniformed mesopores could be generated by using the F127 as the soft template, follow the hyper crosslinking and solvent extraction, the ordered surface intervals could be observed in the final polymeric sheet. The well-defined interval together with the organic functional groups make these polymers effective for carbon capture [49].

Hard template method in principle can be used to prepare any kind of the HCPs, the void size and the shape of the particles are determined by the kinds of hard templates. Therefore, hard template is considered as the most popular and straightforward method. The template can be removed completely and no contributions to the final products, but the toxic etching solvent are often required in the hard template approach for generating of the hollow morphologies. To solve this problem, soft template method also been generated. The interaction between the soft template itself, soft template and catalyst should be firstly predicted, and then we can control the morphologies of the final products by adjusting their interactions, however, sophisticated polymer design should be pay on the soft template preparation. In some approaches, although no need to remove the hard template, but the resultant is organic/inorganic HCP contained hybrid materials. To get the pure morphology-controlled HCP materials, the hard and soft template methods are both inconvenient. 
a
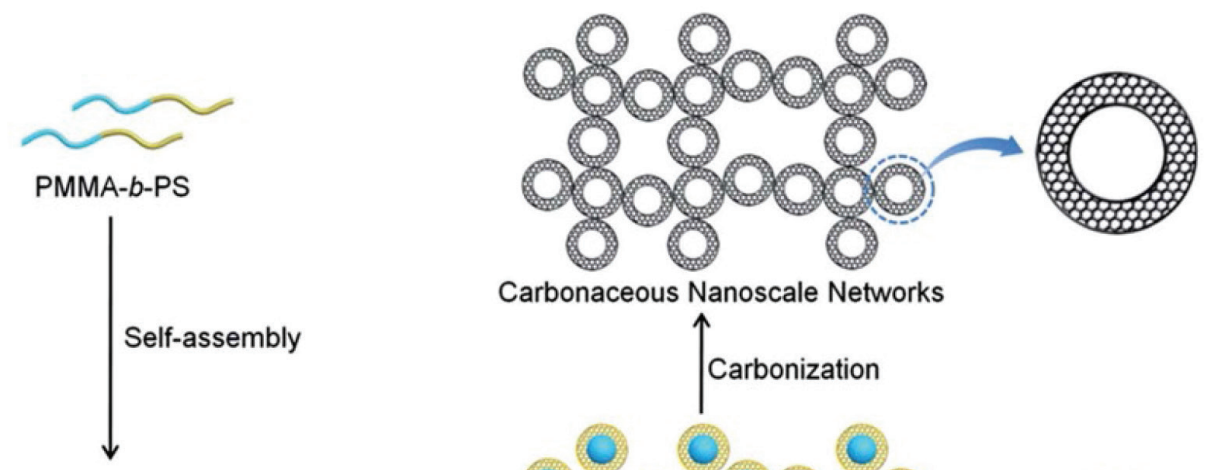

Carbonaceous Nanoscale Networks
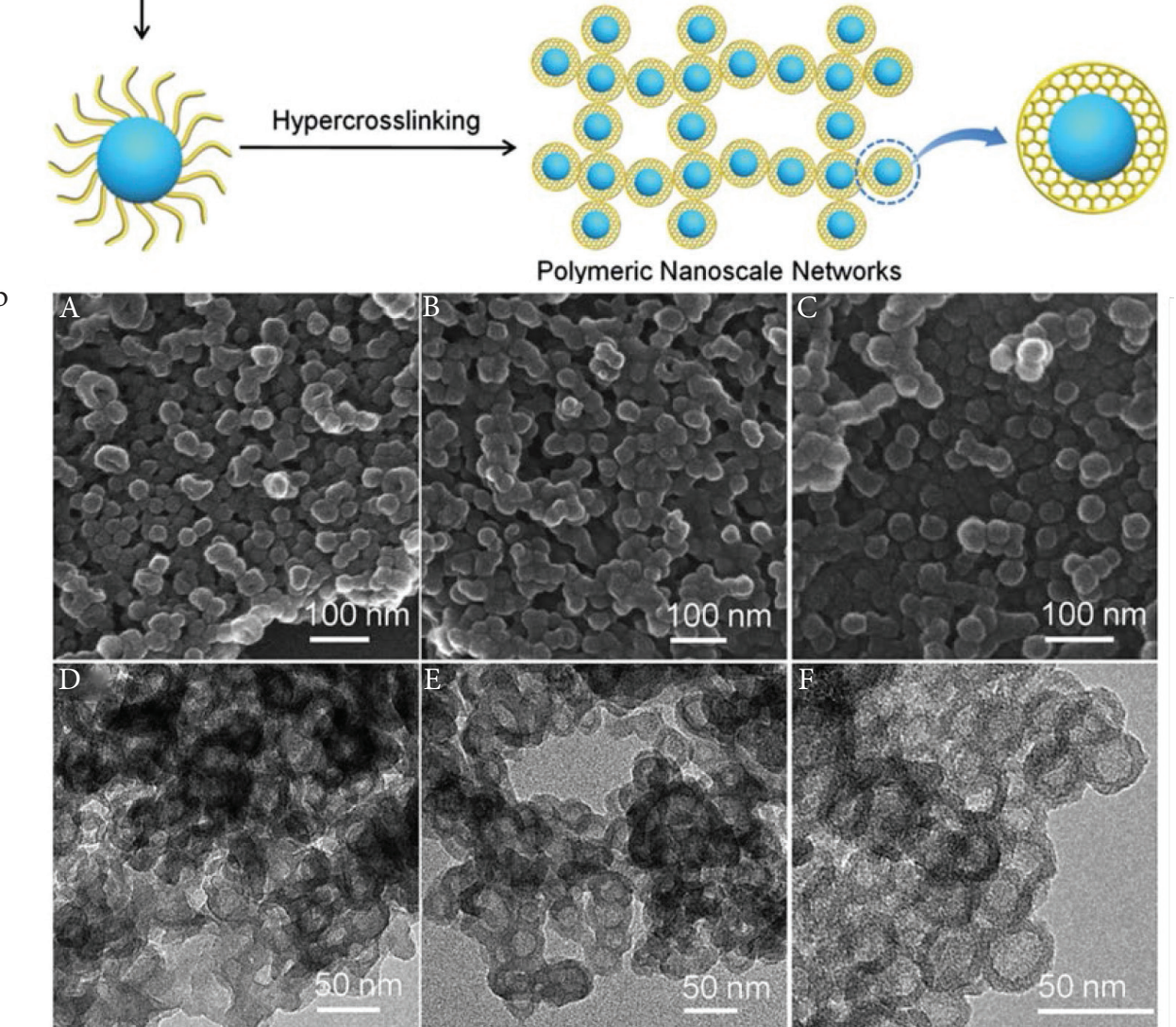

Figure 5 Soft template method for fabricating of the morphology-controlled HCPs. (a) Scheme of preparation of hollow polymer spheres from block copolymer self-assembly and further carbonization. (b) SEM and TEM images of the polymer networks assembled by with different molecular compositions [46]. Copyright 2014. Adapted with permission from the Royal Society of Chemistry.

\subsection{Template Free Method}

In the practical applications, due to the ease to handle, low production cost, and easy for scaling up, synthesizing of the nanomaterials without using any template is preferred. The template-free approaches for generating HCPs morphologies are rarely be reported. The heterogeneous coating or doping, template removal and design are eliminated in these methods, making it more convenient for scale up the quantized product. We guess that these strategies are the frontier and direction for generating the morphology-control of HCPs. The spherical morphologies are the most fundamental shapes in the HCPs area, due to the general nature of $\mathrm{C}$ - $\mathrm{C}$ coupling process, the morphology forming together with the linking of the covalent bonds, which is difficult for controlling the mesopores and macropores, especially the uniform mesoporous morphologies.
The general morphologies by the directly hyper crosslinking polymerization were firstly reported by Cooper et al [7]. The coarser, nodular morphologies are the most situations when subject to the Scanning Electron Microscope (SEM) analysis, and sometime, the HCPs particles without obvious features were also appeared. The sphere is the most fundamental shape that generated by the template-free method. Sherrington et al. synthesized monodisperse porous hyper-crosslinked nanospheres by using the template-free method. Firstly, the gel-type spherical polystyrene precursors with typically $\sim 400 \mathrm{~nm}$ diameter were synthesized by the surfactant-free emulsion polymerization, and then hyper crosslinking were happened between the vinyl group [50]. Huang et al. reported an easy template-free approach to fabricate of the polymeric microspheres. By using the zinc chloride as the Friedel-Crafts catalyst, the chloromethyl methyl ether as the crosslinker agent, and the dichloromethane as the solvent, the 3D interconnected polymeric spheres with the micro-, meso-, and 
macropores formed botryoid networks. Also, these spheres could further be pyrolyzed and formed carbon counterparts, which afford excellent capacitive performance in the supercapacitor test [33]. Jiang et al. found a series of $1 \mathrm{D}$ hypercrosslinked polymer nanotubes could be generated by the $\mathrm{FeCl}_{3}$ catalyzed Friedel-Crafts reaction of the aromatic hydrocarbons. The monomer concentration and mechanical agitation were found play crucial roles in the morphology forming, nevertheless, the formation mechanism is still unclear [20]. After then, Zhang et al. reported a newly developed dual oil phase system to synthesize of the tubular nanofibers. In this new approach, the conventional Dichlorop-xylene (DCX) was firstly dissolved in the 1,2-dichloroethane and further transferred to the polydimethylsiloxane to form an oil phase suspension system. Compare with the traditional reaction system, this system could confine the hyper crosslinking in the oil phase droplet to keep the balance of the system. The author guess DCX or Lewis acid catalysts may form the crystal and act as the self-template simultaneously in the reaction. The substituent position, the number of the substituents, and the type of the substituent halogens all have impact on the final polymer morphologies. The disadvantage of the method is that the Brunner-Emmet-Teller measurements (BET) surface area of the obtained polymers is relatively low [51]. Hu et al. also follow this method to constructed multiple-structured tubular carbon nanofibers@TiO hybrid composite. The desirable design in the microstructures could provide excellent wide-band and lightweight microwave absorbents performance [52].

Not only tubular morphologies, the sheet-liked morphologies also could be generated by the template free method, for example, the Jia et al. using the steric hexakis(benzylthio)benzene and thiophene as comonomers, the chloroform acts as the cross-linker to dictate the cross-linking degree between the two monomers. By simply adjust the solvent amount of chloroform, the morphologies of HCPs could be precisely tailored from nanosheets to nanotubes. The resultant HCPs exhibit excellent iodine uptake capacity and high absorption rate [13]. The adsorption capacity of HCP for iodine reached $270 \%$ and equilibrium rapid adsorption could be achieved within $60 \mathrm{~min}$. As illustrated in Figure 6, Song et al. showed the Lewis acid-base interaction had a dramatic effect on the hyper cross-linking, the self-assembled morphologies could be tailored from nanotubes to hollow spheres and even nanosheets. These polymers could be transfer to acid-functionalized catalyst for the biodiesel production. And the porous carbonaceous counterparts also could be generated by pyrolysis of the corresponding polymer precursors $[13,53,54]$. Also, from Kim et al., their reported the crossing linking agent play a key role on the morphology and porosity design of HCPs. By simply varying monomer to formaldehyde dimethylacetal (FDA) ratio, the diversity HCPs morphologies, such as nanospheres, nanofibers, and flower-shapes could be generated [55]. The high surface area and porous nature make these polymers exhibit outstanding adsorption capacity and pollutant remove ability. And the corresponding carbon counterparts showing outstanding electrochemical performance and stable capacitance retention [56]. Tan et al. also proposed an ultrasonic-assisted solvent exfoliation method for generating of the layered polymers. Unlike other approach by using the FDA as the cross-linking agent, the dichloroalkane are induced to the system to be an economical solvent, stable electrophilic environment, and extra cross-linker. This method demonstrated a new and inexpensive ideas for preparing the morphology-controlled HCPs.

Films are also important materials for the area of separation and purification. The polystyrene-based polymers could be utilized as the HCPs' precursor. Qiao et al. described a strategy for preparing HCP membranes as the precursor, following by the conventional Friedel-Crafts reaction with FDA as the crosslinker. These membranes demonstrate excellent performance for selecting of the small gas molecules [57]. Also, by using the same direct synthetization method, the ionic liquid conjugated HCPs also been fabricated and applied for the $\mathrm{H}_{2}$ generation [58].

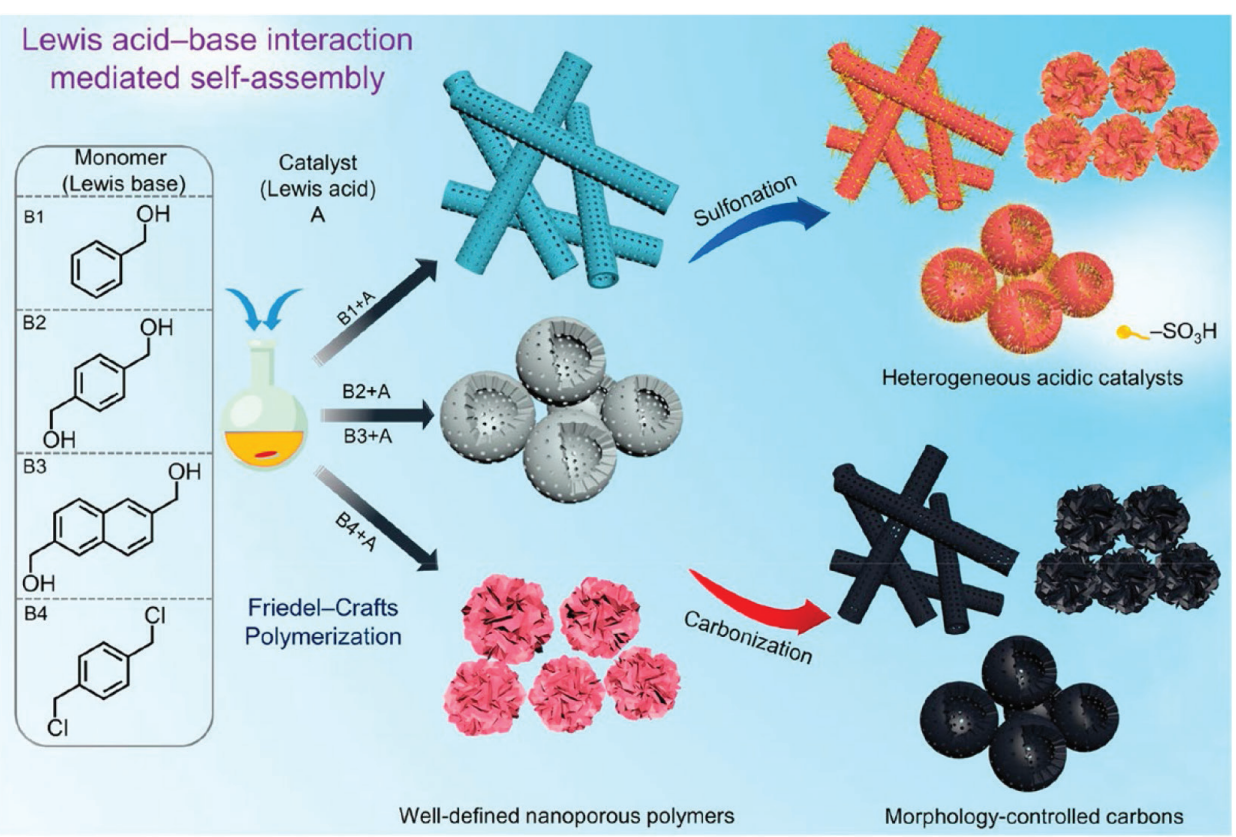

Figure 6 Represented template free method for fabricating of the morphology-controlled HCPs. (a) Scheme of preparation of polymeric nanotubes, hollow polymer spheres and nanosheets. Sulfonation of the resultant HCPs to yield the heterogeneous acidic catalysts, and carbonization of the HCPs to yield the porous morphology-controlled carbon materials [13]. Copyright 2019. Adapted with permission from the American Chemical Society. 
The template free method solves the problem existed on the templating synthesis; the tedious, time-consuming procedure are not required. However, the exquisite choosing of the assembly staring materials seems particularly important. In the template free method, understanding the role of the monomer catalysts and the cross-linking agent seems extremely important for synthesizing of the morphology-controlled HCPs.

\subsection{Physical-Mechanical Method}

Beside of the template and template-free method, the physical and mechanical methods are also reported as the useful solution for assembling of the HCPs. Electro spin is the useful method for synthesizing of the fiber-liked HCPs. Wu et al. firstly apply this method into the HCPs area, the stable continuous nanofibers was fabricated by the electrospinning process and collected by the aluminum foil. The stepwise crosslinking was applied two times by the conventional Friedel-Crafts knitting method [59]. Also, recently, Szekely reported the robust nanofibrous adsorptive membranes were fabricated via electrospinning, after crosslinked with the graphitic acid linker, the robust covalent polymer networks were generated (Figure 7). The nanofibrous membrane could be used for rapid and efficient scavenging of the organic pollutants [60]. Recently, Horike also report a green, low-cost, and mechanochemical route to synthesize the HCPs with the relatively high surface of $782 \mathrm{~m}^{2} / \mathrm{g}$ in only $5 \mathrm{~min}$. A variety of aromatic molecules could be used as the monomer for synthesizing of the highly porous polymers within minutes [61].

a

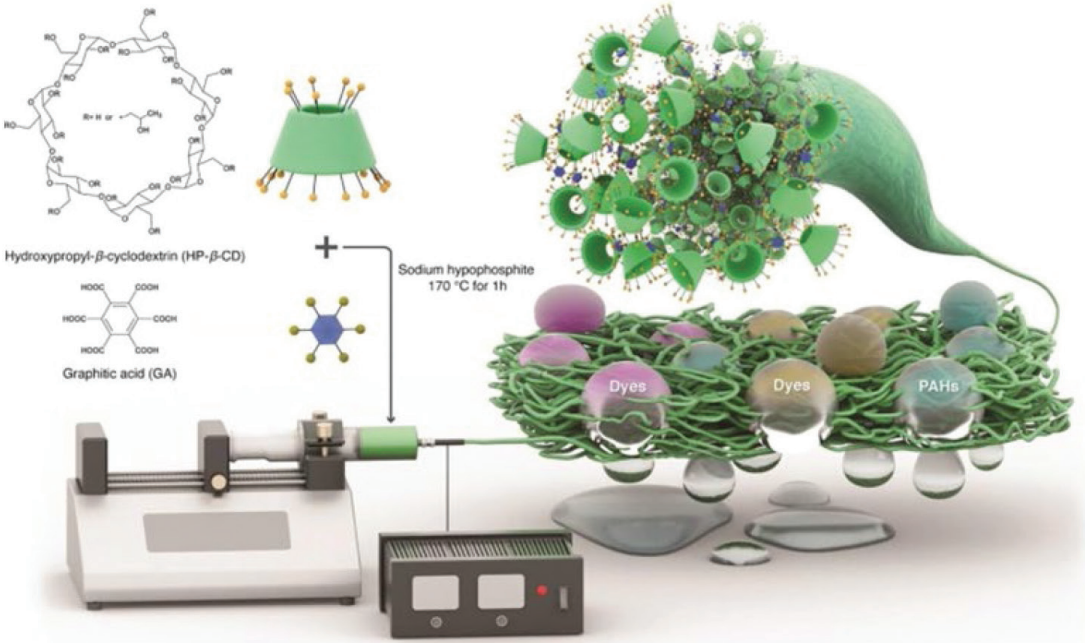

$\mathrm{b}$
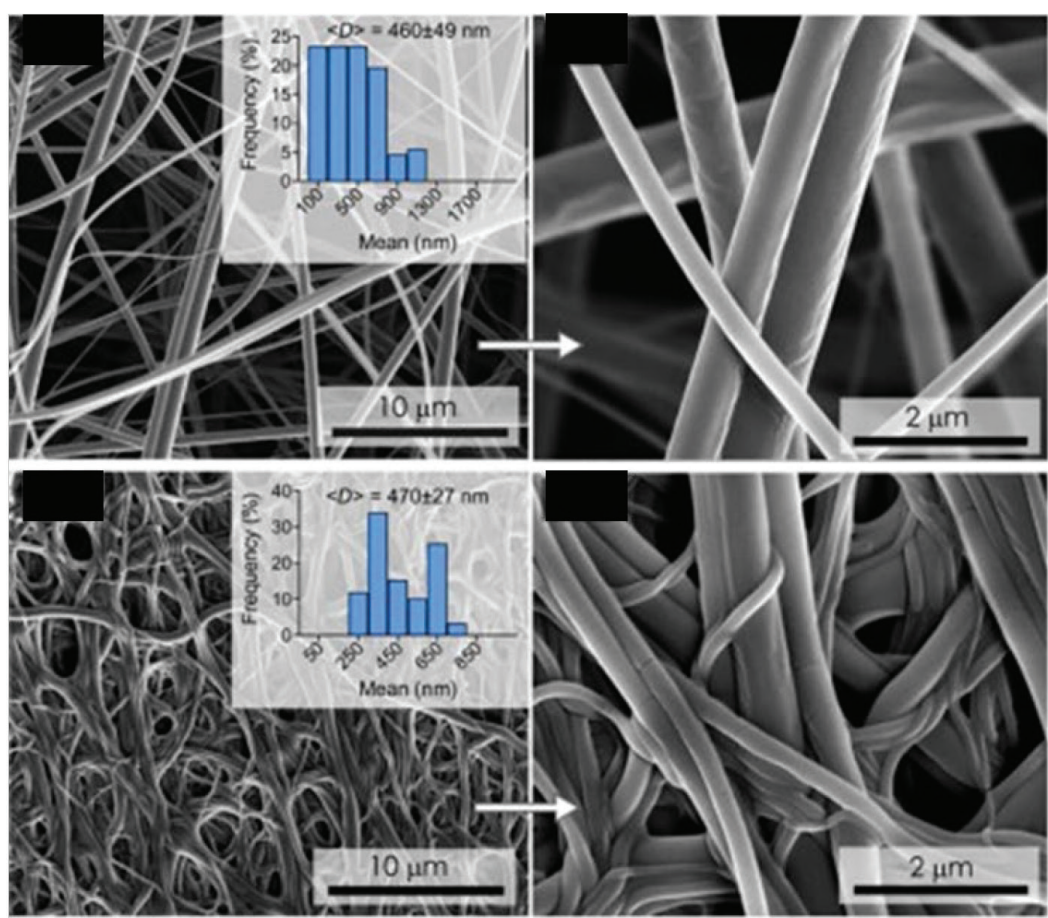

Figure 7 The electro spin was used as the tool for generating of the hyper crosslinked cyclodextrin networks. (a) Scheme of fabrication the fiber-liked HCPs and the scavenging of organic pollutants. (b) SEM images of the hypercrosslinked polymers [60]. Copyright 2021. Adapted with permission from the Elsevier. 


\section{CONCLUSION AND FUTURE CHALLENGES}

The design, synthesis, and application of hypercrosslinked polymers with micro-, meso- and macroporous structures occupies at a very important position in the field of materials science. Hyper cross-linking polymers mainly based on Friedel-Crafts chemistry, the $\mathrm{C}-\mathrm{C}$ bond quickly formation during the reaction and resulting a highly cross-linked network. This polymerization route makes it difficult to obtain porous polymers with controlled morphologies in one step.

Three main approaches, including of templated, templated-free and physical-mechanical method, are induced to the HCPs synthetization Diverse interesting morphologies including of the spheres, hollow spheres, fibers, sheets and membranes could be generated by reported methods. The pore distribution can be concentrated from the micro to the meso- and macropore regions, and even has hierarchical pore distribution by varying of the morphologies. The ordered pore channel and uniform shapes as an excellent platform for catalysis and adsorption. The special morphologies such as the hollow spheres offer the platform for storage the drug and controlled release, also could be used for nanocarrier and nanoreactor. The various void in the layered structures could easily anchor the metals. Meanwhile, the HCPs generally have the high surface area and excellent stability. Thus, the morphology-controlled HCPs could play an important role for the task-specific applications and improving the application performance.

In this review, we summarize the recent development in HCPs synthetization, especially for controlling the morphologies. For the comprehensive understanding, we classified these methods into three main approach, and we have discussed in detail and compared the templated, templated-free, and physical-mechanical method for generating the morphology-controlled HCPs. For the hard templated method, providing the templates with more dimensions and scales is the most important future direction. For the soft-template method, quickly preparing the soft templates and finding the corresponding assembly parameter is the decisive factors. While for the template-free method, wisely choosing the monomers, catalysts, solvents, and cross-linking agents become the key for assembling of the HCPs, this means we should clearly understand the interactions between these parameters. In the physical-mechanical method, the sophisticated design of the machine and spray nozzle is as important as the selection of the staring materials. It should be mentioned that this research area is developing and that this review is not exhaustive as new studies are reported quite frequently and the limitation of our available documents. Continued efforts should be pay on simplify the steps and mass production by utilizing of the environmental beginning strategy.

\section{CONFLICTS OF INTEREST}

The authors declare they have no conflicts of interest.

\section{AUTHORS’ CONTRIBUTION}

WS contributed in conceptualization, software, writing (original draft) and review and editing the manuscript and also in funding acquisition. MZ contributed in writing (review and editing) the manuscript. ML and YH contributed in writing (original draft) the manuscript. DY contributed in conceptualization, supervision and funding acquisition.

\section{ACKNOWLEDGMENT}

This work was supported by the Shanghai Sailing Program (21YF1431000).

\section{REFERENCES}

[1] Tan L, Tan B. Hypercrosslinked porous polymer materials: design, synthesis, and applications. Chem Soc Rev 2017;46:3322-56.

[2] Chaoui N, Trunk M, Dawson R, Schmidt J, Thomas A. Trends and challenges for microporous polymers. Chem Soc Rev 2017;46:3302-21.

[3] Huang J, Richard Turner S. Hypercrosslinked polymers: a review. Polym Rev 2018;58:1-41.

[4] Abbott LJ, Colina CM. Formation of microporosity in hypercross-linked polymers. Macromolecules 2014;47:5409-15.

[5] Li X, Chen G, Jia Q. One-pot synthesis of viologen-based hypercrosslinked polymers for efficient volatile iodine capture. Microporous Mesoporous Mater 2019;279:186-92.

[6] Zeng X, Huang J. Anisole-modified hyper-cross-linked resins for efficient adsorption of aniline from aqueous solution. J Colloid Interface Sci 2020;569:177-83.

[7] Wood CD, Tan B, Trewin A, Niu H, Bradshaw D, Rosseinsky MJ, et al. Hydrogen storage in microporous hypercrosslinked organic polymer networks. Chem Mater 2007;19:2034-48.

[8] Bhunia S, Banerjee B, Bhaumik A. A new hypercrosslinked supermicroporous polymer, with scope for sulfonation, and its catalytic potential for the efficient synthesis of biodiesel at room temperature. Chem Commun 2015;51:5020-3.

[9] Gu Y, Son SU, Li T, Tan B. Low-cost hypercrosslinked polymers by direct knitting strategy for catalytic applications. Adv Funct Mater 2021;31:2008265.

[10] Bi J, Dong Y, Zhu D, Guo W, Meng D, Li T. Pd Cu decorate hypercrosslinked network: synthesis and application as efficient catalysts for the reduction of 4-nitrophenol and Suzuki-Miyaura coupling reaction. Appl Surf Sci 2019;495:143584.

[11] Bai J, Zhang W, Ma X, Chen L, Liu L, Zhang C. Synthesis of novel hyper-cross-linked chiral porous polymers and their applications in enantioselective adsorption of amino acids. Microporous Mesoporous Mater 2020;294:109892.

[12] Ma K, Cui H, Zhou A, Wu H, Dong X, Zu F, et al. Mesoporous hydroxyapatite: synthesis in molecular self-assembly and adsorption properties. Microporous Mesoporous Mater 2021;323: 111164.

[13] Li X, Xiong H, Jia Q. A versatile solvent-induced polymerization strategy to synthesize free-standing porous polymer nanosheets and nanotubes for fast iodine capture. ACS Appl Mater Interfaces 2019;11:46205-11.

[14] Zhou L, Chai K, Yao X, Ji H. Enhanced recovery of acetophenone and 1-phenylethanol from petrochemical effluent by highly porous starch-based hypercrosslinked polymers. Chem Eng J 2021;418:129351.

[15] Vinodh R, Muralee Gopi CVV, Kummara VGR, Atchudan R, Ahamad T, Sambasivam S, et al. A review on porous carbon 
electrode material derived from hypercross-linked polymers for supercapacitor applications. J Energy Storage 2020; 32:101831.

[16] Wang H, Li Z, Meng Z, Guo X, Du Y, Yang H. An easily obtained hypercrosslinked pyrene-based porous organic polymer as a high-performance electrode material for lithium-ion batteries. New J Chem 2021;45:7060-4.

[17] Deka N, Barman J, Kasthuri S, Nutalapati V, Dutta GK. Transforming waste polystyrene foam into N-doped porous carbon for capacitive energy storage and deionization applications. Appl Surf Sci 2020;511:145576.

[18] Li Q, Razzaque S, Jin S, Tan B. Morphology design of microporous organic polymers and their potential applications: an overview. Sci China Chem 2017;60:1056-66.

[19] Zhuang X, Gehrig D, Forler N, Liang H, Wagner M, Hansen MR, et al. Conjugated microporous polymers with dimensionalitycontrolled heterostructures for green energy devices. Adv Mater 2015;27:3789-96.

[20] Wang X, Mu P, Zhang C, Chen Y, Zeng J, Wang F, et al. Control synthesis of tubular hyper-cross-linked polymers for highly porous carbon nanotubes. ACS Appl Mater Interfaces 2017;9:20779-86.

[21] Jang JY, Le TMD, Ko JH, Ko YJ, Lee SM, Kim HJ, et al. Triple-, double-, and single-shelled hollow spheres of sulfonated microporous organic network as drug delivery materials. Chem Mater 2019;31:300-4.

[22] Ryu SH, Lee DH, Lee SM, Kim HJ, Ko YJ, Ko KC, et al. Morphology engineering of a suzuki coupling-based microporous organic polymer (MOP) using a sonogashira coupling-based MOP for enhanced nitrophenol sensing in water. Chem Commun 2019;55:9515-18.

[23] Wang J, Cui Y, Wu F, Shah T, Ahmad M, Zhang A, et al. Coreshell structured $\mathrm{Fe} / \mathrm{Fe}_{3} \mathrm{O}_{4} @ \mathrm{TCNFS} @ \mathrm{TiO}_{2}$ magnetic hybrid nanofibers: preparation and electromagnetic parameters regulation for enhanced microwave absorption. Carbon 2020;165:275-85.

[24] He Z, Zhou M, Wang T, Xu Y, Yu W, Shi B, et al. Hyper-crosslinking mediated self-assembly strategy to synthesize hollow microporous organic nanospheres. ACS Appl Mater Interfaces 2017;9:35209-17.

[25] Wu D, Xu F, Sun B, Fu R, He H, Matyjaszewski K. Design and preparation of porous polymers. Chem Rev 2012;112: 3959-4015.

[26] Wang S, Shao L, Sang Y, Huang J. Hollow hyper-cross-linked polymer microspheres for efficient rhodamine B adsorption and $\mathrm{CO}_{2}$ capture. J Chem Eng Data 2019;64:1662-70.

[27] Li B, Yang X, Xia L, Majeed MI, Tan B. Hollow microporous organic capsules. Sci Rep 2013;3:2128.

[28] Li Q, Jin S, Tan B. Template-mediated synthesis of hollow microporous organic nanorods with tunable aspect ratio. Sci Rep 2016;6:31359.

[29] Zhao W, Hou Z, Yao Z, Zhuang X, Zhang F, Feng X. Hypercrosslinked porous polymer nanosheets: 2D RAFT agent directed emulsion polymerization for multifunctional applications. Polym Chem 2015;6:7171-8.

[30] Xu S, Weng Z, Tan J, Guo J, Wang C. Hierarchically structured porous organic polymer microspheres with built-in $\mathrm{Fe}_{3} \mathrm{O}_{4}$ supraparticles: construction of dual-level pores for Pt-catalyzed enantioselective hydrogenation. Polym Chem 2015;6:2892-9.

[31] Rathod PB, Pandey AK, Meena SS, Athawale AA. Quaternary ammonium bearing hyper-crosslinked polymer encapsulation on $\mathrm{Fe}_{3} \mathrm{O}_{4}$ nanoparticles. RSC Adv 2016;6:21317-25.
[32] He J, Razzaque S, Jin S, Hussain I, Tan B. Efficient synthesis of ultrafine gold nanoparticles with tunable sizes in a hyper-crosslinked polymer for nitrophenol reduction. ACS Appl Nano Mater 2019;2:546-53.

[33] Huang X, Kim S, Heo MS, Kim JE, Suh H, Kim I. Easy synthesis of hierarchical carbon spheres with superior capacitive performance in supercapacitors. Langmuir 2013;29:12266-74.

[34] Razzaque S, Wang K, Hussain I, Tan B. Facile synthesis of hypercrosslinked hollow microporous organic capsules for electrochemical sensing of $\mathrm{Cu}^{\mathrm{II}}$ ions. Chem Eur J 2019;25:548-55.

[35] Razzaque S, Cai C, Lu QW, Huang FZ, Li YS, Tang HB, et al. Development of functionalized hollow microporous organic capsules encapsulating morphine - an in vitro and in vivo study. J Mater Chem B 2017;5:742-9.

[36] Jia Z, Wang K, Tan B, Gu Y. Hollow hyper-cross-linked nanospheres with acid and base sites as efficient and waterstable catalysts for one-pot tandem reactions. ACS Catal 2017; 7:3693-702.

[37] Choi SJ, Choi EH, Song C, Ko YJ, Lee SM, Kim HJ, et al. Hypercross-linked polymer on the hollow conjugated microporous polymer platform: a heterogeneous catalytic system for poly (caprolactone) synthesis. ACS Macro Lett 2019;8:687-93.

[38] Yang X, Song K, Tan L, Hussain I, Li T, Tan B. Hollow microporous organic capsules loaded with highly dispersed Pt nanoparticles for catalytic applications. Macromol Chem Phys 2014;215: 1257-63.

[39] Wu D, Hui CM, Dong H, Pietrasik J, Ryu HJ, Li Z, et al. Nanoporous polystyrene and carbon materials with core-shell nanosphereinterconnected network structure. Macromolecules 2011;44: 5846-9.

[40] Mai W, Sun B, Chen L, Xu F, Liu H, Liang Y, et al. Waterdispersible, responsive, and carbonizable hairy microporous polymeric nanospheres. J Am Chem Soc 2015;137:13256-9.

[41] Xie Y, Huang W, Zheng B, Li S, Liu Q, Chen Z, et al. All-in-one porous polymer adsorbents with excellent environmental chemosensory responsivity, visual detectivity, superfast adsorption, and easy regeneration. Adv Mater 2019;31:e1900104.

[42] Huang K, Rzayev J. Well-defined organic nanotubes from multicomponent bottlebrush copolymers. J Am Chem Soc 2009; 131:6880-5.

[43] Zhang H, Xiong L, He Z, Zhong A, Wang T, Xu Y, et al. Functionalized microporous organic nanotube networks as a new platform for highly efficient heterogeneous catalysis. Polym Chem 2016;7:4975-82.

[44] Meng G, Gao S, Liu Y, Zhang L, Song C, Huang K. Amino- and sulfo-bifunctionalized hyper-crosslinked organic nanotube frameworks as efficient catalysts for one-pot cascade reactions. New J Chem 2019;43:2269-73.

[45] Zhang H, Zhou M, Xiong L, He Z, Wang T, Xu Y, et al. Oxovanadium (IV) complex supported by microporous organic nanotube frameworks: a high selective heterogeneous catalyst for the oxidation of thiols to disulfides. Microporous Mesoporous Mater 2018;255:103-9.

[46] Li Z, Wu D, Huang X, Ma J, Liu H, Liang Y, et al. Fabrication of novel polymeric and carbonaceous nanoscale networks by the union of self-assembly and hypercrosslinking. Energy Environ Sci 2014;7:3006-12.

[47] Gao TN, Wang T, Wu W, Liu Y, Huo Q, Qiao ZA, et al. Solventinduced self-assembly strategy to synthesize well-defined hierarchically porous polymers. Adv Mater 2019;31:1806254. 
[48] Wang T, Xu Y, He Z, Zhou M, Yu W, Shi B, et al. Fabrication of sulphonated hollow porous nanospheres and their remarkably improved catalytic performance for biodiesel synthesis. React Funct Polym 2018;132:98-103.

[49] Zhang J, Qiao ZA, Mahurin SM, Jiang X, Chai SH, Lu H, et al. Hypercrosslinked phenolic polymers with well-developed mesoporous frameworks. Angew Chem Int Ed Engl 2015;54: 4582-6.

[50] Macintyre FS, Sherrington DC, Tetley L. Synthesis of ultrahigh surface area monodisperse porous polymer nanospheres. Macromolecules 2006;39:5381-4.

[51] Wang J, Yang Z, Ahmad M, Zhang H, Zhang Q, Zhang B. A novel synthetic method for tubular nanofibers. Polym Chem 2019;10: 4239-45.

[52] Kang S, Qiao S, Cao Y, Hu Z, Yu J, Wang Y, et al. Hyper-crosslinked polymers-derived porous tubular carbon nanofibers@ $\mathrm{TiO}_{2}$ toward a wide-band and lightweight microwave absorbent at a low loading content. ACS Appl Mater Interfaces 2020;12: 46455-65.

[53] Song W, Zhang Y, Varyambath A, Kim I. Guided assembly of well-defined hierarchical nanoporous polymers by lewis acidbase interactions. ACS Nano 2019;13:11753-69.

[54] Song W, Zhang Y, Varyambath A, Kim JS, Kim I. Sulfonic acid modified hollow polymer nanospheres with tunable wall-thickness for improving biodiesel synthesis efficiency. Green Chem 2020;22:3572-83.
[55] Varyambath A, Song W, Singh S, Kim JS, Kim I. Tunable construction of biphenyl-based porous polymeric nanostructures and their synergistically enhanced performance in pollutant adsorption and energy storage. Microporous Mesoporous Mater 2021;312:110800.

[56] Wang S, Zhang C, Shu Y, Jiang S, Xia Q, Chen L, et al. Layered microporous polymers by solvent knitting method. Sci Adv 2017;3:e1602610.

[57] Qiao ZA, Chai SH, Nelson K, Bi Z, Chen J, Mahurin SM, et al. Polymeric molecular sieve membranes via in situ cross-linking of non-porous polymer membrane templates. Nat Commun 2014;5:3705.

[58] Chinnappan A, Chung WJ, Kim H. Hypercross-linked microporous polymeric ionic liquid membranes: synthesis, properties and their application in $\mathrm{H}_{2}$ generation. J Mater Chem A 2015;3:22960-8.

[59] Liu H, Li S, Yang H, Liu S, Chen L, Tang Z, et al. Stepwise crosslinking: a facile yet versatile conceptual strategy to nanomorphology-persistent porous organic polymers. Adv Mater 2017;29:1700723.

[60] Topuz F, Holtzl T, Szekely G. Scavenging organic micropollutants from water with nanofibrous hypercrosslinked cyclodextrin membranes derived from green resources. Chem Eng J 2021; 419:129443.

[61] Lee JSM, Kurihara T, Horike S. Five-minute mechanosynthesis of hypercrosslinked microporous polymers. Chem Mater 2020;32: 7694-702. 